\title{
IMPROVING THE COST PERFORMANCE OF MECHANICAL ELECTRICAL AND PLUMBING (MEP) WORKS IN HOTEL BUILDINGS BASED ON BUILDING INFORMATION MODELING (BIM) 5D
}

\author{
Albert Eddy Husin ${ }^{1 *}$, Syamsir Abe Sihombing ${ }^{1}$, Bernadette Detty Kussumardianadewi ${ }^{1}$, Diah \\ Ika Rahmawati ${ }^{1}$
}

${ }^{1}$ Department of Civil Engineering, Universitas Mercu Buana, Jakarta Barat 11650, Indonesia

(Received: October 2020 / Revised: November 2020 / Accepted: December 2020)

\begin{abstract}
High-rise building projects expose a high risk due to structural design complexity, large workloads, and long project duration. The complexity of the work includes the design of Mechanical, Electrical, Plumbing (MEP), HVAC (Heating, Ventilation and Air Conditioning) systems, early warning, watering systems, hydrants, sprinklers, evacuation routes in the form of emergency stairs and fire doors. This research focuses on the application of BIM 5D to estimate and improve the cost performance of MEP work in high-rise hotel buildings. This research combines qualitative and quantitative approaches through in-depth interviews and BIM 5D modelling to achieve the research objective. The results showed influential factors for BIM implementation namely 2D Drawings, BIM 5D implementation, specification and technical plan, operator experiences, BIM 5D models, individual selection model, estimating, calculation process, cost database, and operator education. The findings of this research also show a cost efficiency of $3.56 \%$ from the BIM 5D implementation to the high-rise hotel building.
\end{abstract}

Keywords: Hotel High-Rise Building; Mechanical, Electrical, and Plumbing (MEP); Building Information Modeling (BIM) 5D; Cost Efficiency

\section{INTRODUCTION}

High-rise building projects expose a high risk due to structural design complexity, large workloads, and long project duration (Abu Hammad et al., 2010; Aneziris et al., 2012). The complexity of the work includes the design of the following: mechanical, electrical, and plumbing (MEP); heating, ventilation and air conditioning (HVAC); early warning; watering systems; hydrants; sprinklers; evacuation routes in the form of emergency stairs; and fire doors designed according to needs evacuation. MEP is a working system in high-rise buildings in mechanical, electrical control, plumbing systems, which include sewage/wastewater (dirty and used water), disposal systems, venting systems, rainwater, and clean water supply. The percentage of this work for the total construction work of high-rise building projects is equal to $26 \%$ (Riley et al., 2005; Wang \& Leite, 2016).

In order to achieve the project success, building's stakeholder needs to consider current technology such as Building Information Modeling (BIM) system. This technology aims to identify the problem in terms of structural, architectural, MEP work during project operation and maintenance and detect failure at an early stage (Bynum et al., 2013; Volk et al., 2014).

\footnotetext{
*Corresponding author's email: albert_eddy@mercubuana.ac.id 
BIM is a set of technology, whose entire process runs in a digital model using an integrated and involve a three-dimensional image. BIM uses 3D, real-time, and dynamic modeling software to increase productivity in building design and construction. This technology also involves generating and managing construction data during its life cycle. Previous research has shown that BIM estimation may reduce time and error, in addition, to improve estimation performance when compared to traditional estimation practices (Barlish \& Sullivan, 2012; Husin, Setyawan, et al., 2019; Staub-French et al., 2003).

Currently, BIM technology has been developed into an advanced form of Five-Dimensional Building Information Modelling (BIM-5D). BIM-5D can assist in calculating the volume of work, including overlapping MEP work in one job against another. By modeling the Building Revit on BIM 5D, the complexity of the MEP work can be easier in calculating the volume that affects the financing. MEP works present a diverse and complex system of data collection and calculation of cost estimates (Alrashed \& Kantamaneni, 2018; Charef et al., 2018; Husin, Fahmi, et al., 2019).

The use of the software through the collaboration of BIM 5D is expected to provide faster and more accurate quantification in the planning or design of MEP. In reality, contractors often found limited accuracy between the planning and execution stage. This inaccuracy in estimating volume and price calculation of the cost budget in MEP work may relate to the short duration for quantification during the planning stage (Akhil \& Das, 2019; Azhar et al., 2011). In the longerterm, this has a major effect on the calculation of costs or the Budget Plan for the MEP work in terms of cost overruns and materials waste for the whole project.

Subsequently, this study focuses on the application of BIM 5D to estimate and improve the cost performance of MEP work in high-rise hotel buildings. The research also attempts to determine the percentage cost allocated for MEP work from the overall cost of architectural work, structural work, interior work, lighting work, and landscape work.

\section{METHODS}

This study combines qualitative and quantitative approaches through in-depth interviews and BIM-based analysis :

Table 1. Data of case study

\begin{tabular}{ll}
\hline \multicolumn{1}{c}{ Data } & \multicolumn{1}{c}{ Explanation } \\
\hline Location & Bumi Serpong Damai, South Tangerang \\
Function & A high-rise Hotel Building \\
Number of Layer & $6^{\text {th }}$ floor \\
Construction Period & 2019 \\
\hline
\end{tabular}

In-depth interviews were conducted through questionnaire surveys with executives and field supervisors in the MEP work of high-rise hotel buildings. The results of the respondent's data were then analyzed by ranking the variables based on the scale of importance using The Relative Importance Index (RII). A previous study argued that the RII method can determine the relative importance of the various causes of delay (Alaloul et al., 2016; Gündüz et al., 2013). RII determines the most influential factors with a ranking system based on the weight of the scores given by respondents after filling out the questionnaire. The descriptive analysis is also used to provide an overview of data characteristics and inferential statistical methods to conclude the data to more general conditions. 


\section{RESULTS AND DISCUSSION}

\subsection{Factors that Most Influence the Implementation of BIM 5D MEP Works}

The results of the RII found the most influential factors based on the weight of the value given by the respondents after filling out the questionnaire. Drawing is the factor that highly contributes to the success of BIM 5D implementation with 0,9951 of RII value. It is followed by BIM 5D implementation, specification and technical plan, operator experiences, BIM 5D models, individual selection model, estimating, calculation process, cost database, and operator education. The results of the RII analysis can be seen in Table 2 .

Table 2. Ten most influential sub factors

\begin{tabular}{clr}
\hline Rank & \multicolumn{1}{c}{ Sub Factors } & RII Index Value \\
\hline 1 & 2D Drawing & 0,9951 \\
2 & BIM 5D implementation & 0,9902 \\
3 & Specification and Technical Plan & 0,9854 \\
4 & Operator Experiences & 0,9805 \\
5 & BIM 5D Models & 0,9756 \\
6 & Individual Selection Model & 0,9707 \\
7 & Estimating & 0,9659 \\
8 & Calculation Process & 0,9610 \\
9 & Cost Database & 0,9561 \\
10 & Operator Education & 0,9512 \\
\hline
\end{tabular}

\subsection{Application of BIM 5D to MEP Work}

\subsubsection{Estimating BIM 5D}

The results of the calculation will be compared with previous studies that stated the potential savings of BOM into the construction project ranges between 6\% to 9\% (Kim et al., 2019). To conduct BIM 5D estimation, a 3D BIM model is first made by taking into account a 2D design drawing and field data. Surveys and measurements in the field are carried out to accurately portray them into the model. It is important to create an individual material model (Individual Model Object) of each material for MEP work to describe the volume and price.

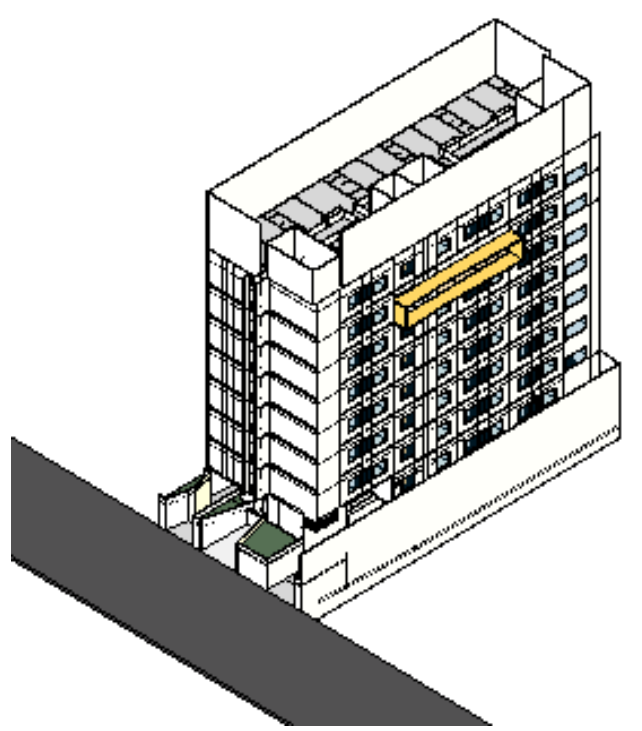

(Revit Architecture view)

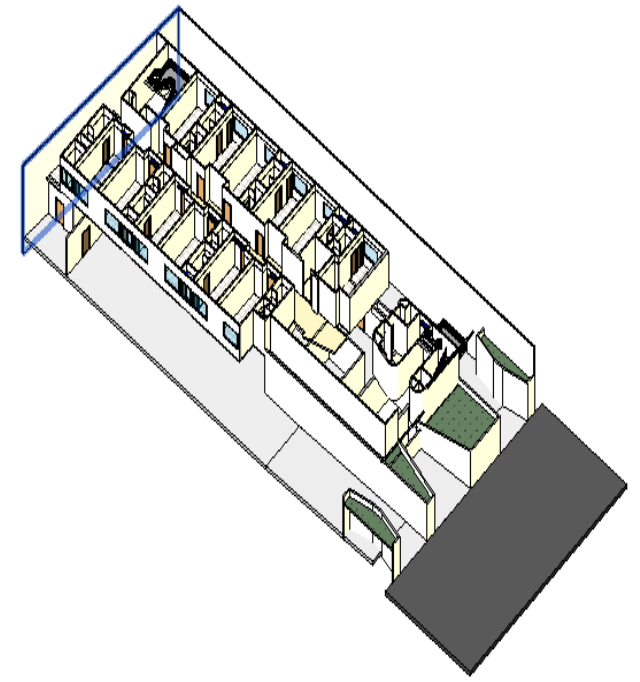

(Display Filter Settings for BIM 5D) 


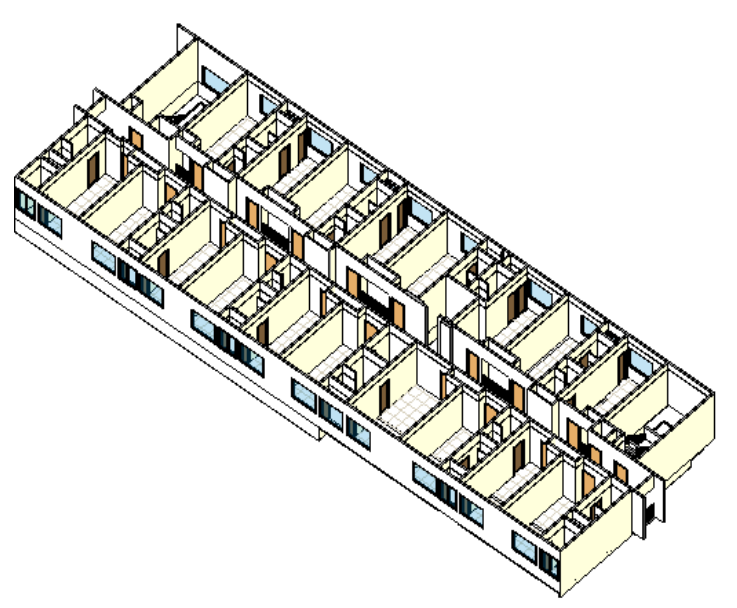

$\left(1^{\text {St }}\right.$ Floor $)$

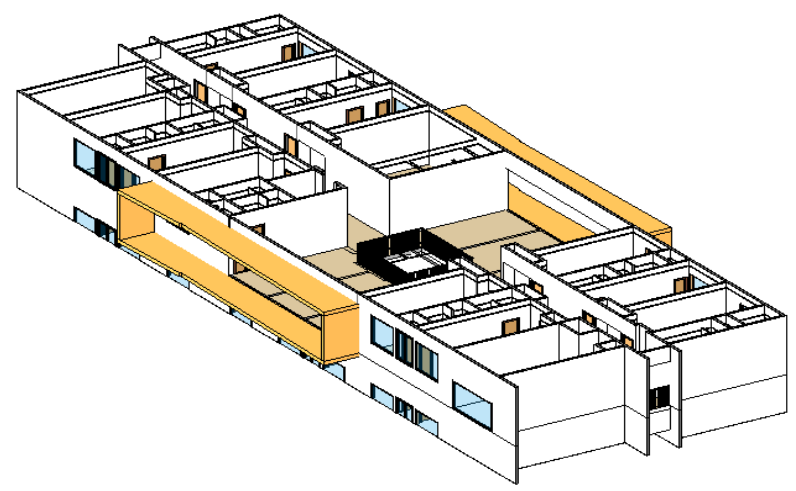

Figure 3 Revit image display

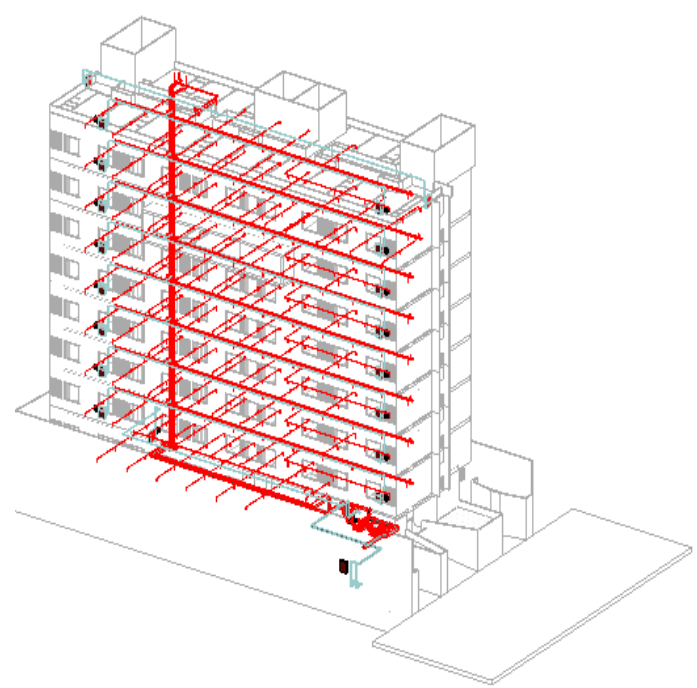

(Fire Hydrant)

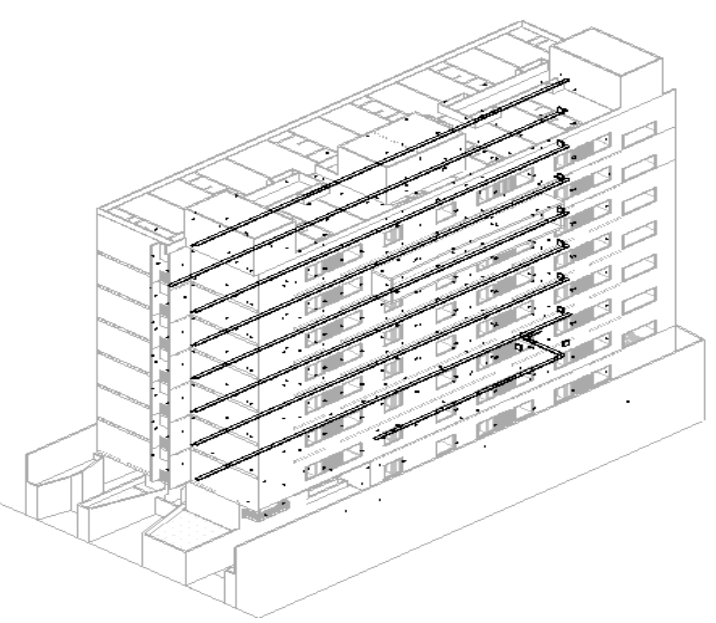

(Electric socket)

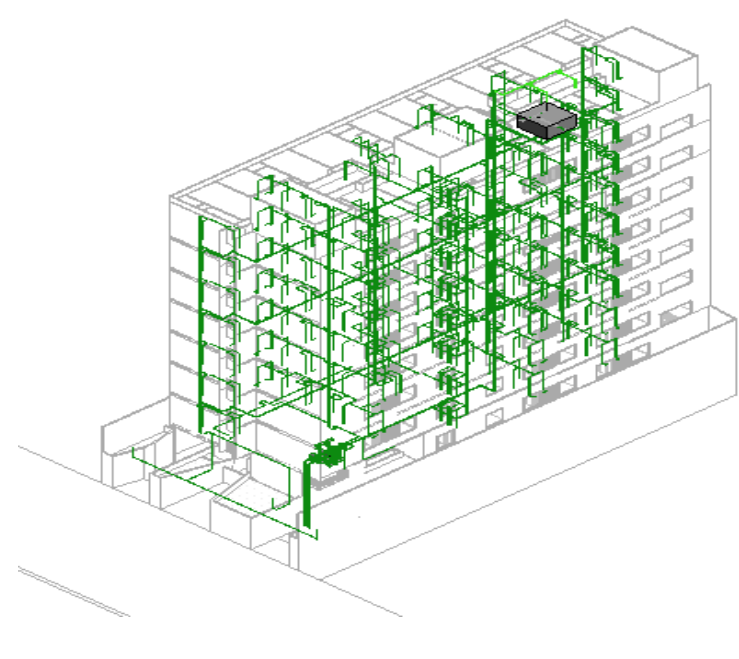

(Plumbing Installation)

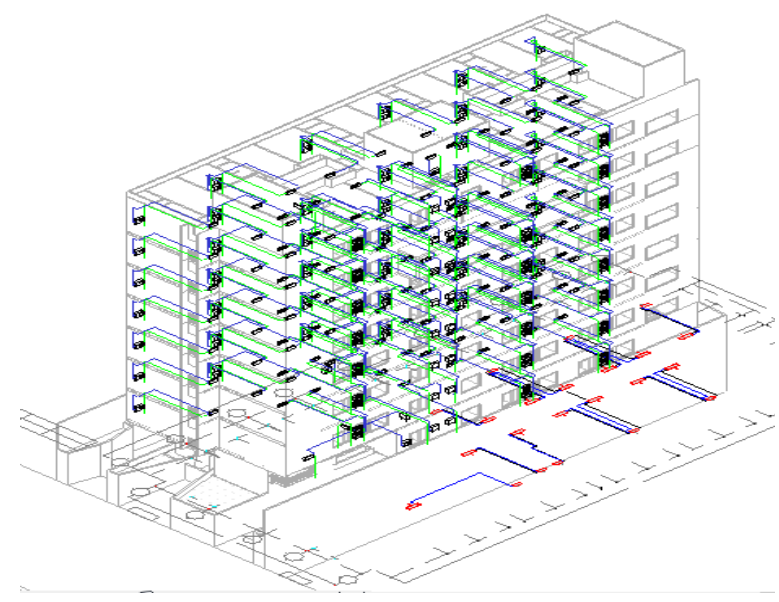

(VAC Plumbing) 


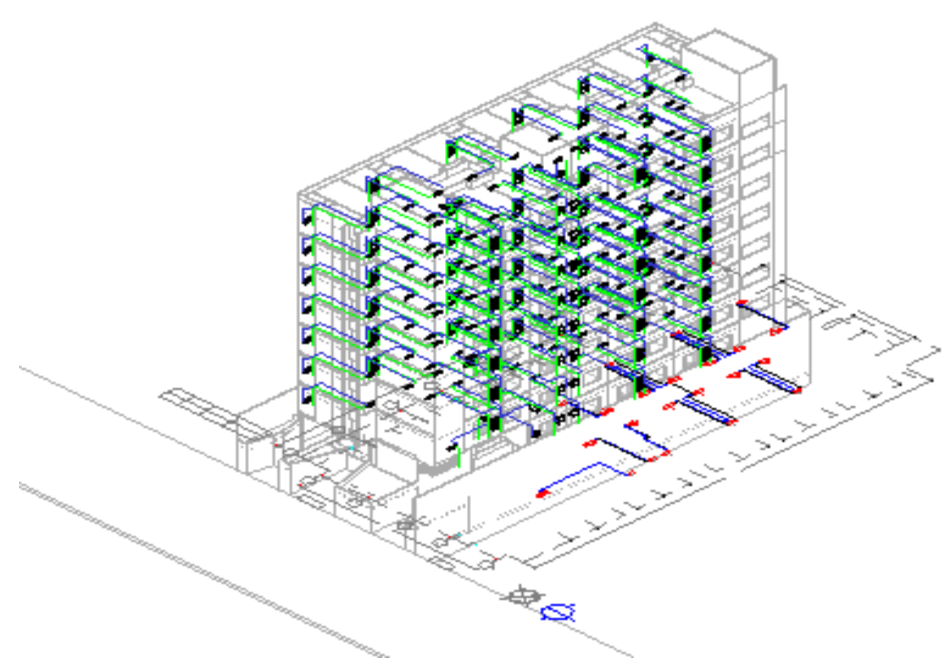

( VAC)

Figure 5. Mechanical, Electrical, and Plumbing Systems

\subsubsection{Volume Calculation}

The steps to carry out BIM 5D begin by selecting the "Analyze" menu in the "Revit 2018" program and then use Report menu and the Schedule / Quantities menu.

Table 3. Result of adding the column

\begin{tabular}{l|c|c|r|r|c}
\hline \multicolumn{7}{c|}{ Type } & Width & Height & Length (m) & Cost & Level \\
\hline Channel Cable Tray & $300 \mathrm{~mm}$ & $100 \mathrm{~mm}$ & 36 & & 1st Floor \\
\hline Subtotal & & & 36 & & \\
\hline Channel Cable Tray & $300 \mathrm{~mm}$ & $100 \mathrm{~mm}$ & 35.9 & & 2nd,3rd,4th,5th,6th,8th Floors \\
\hline Channel Cable Tray & $300 \mathrm{~mm}$ & $100 \mathrm{~mm}$ & 35.9 & & 2nd,3rd,4th,5th,6th,8th Floors \\
\hline Channel Cable Tray & $300 \mathrm{~mm}$ & $100 \mathrm{~mm}$ & 35.9 & & 2nd,3rd,4th,5th,6th,8th Floors \\
\hline Channel Cable Tray & $300 \mathrm{~mm}$ & $100 \mathrm{~mm}$ & 35.9 & & 2nd,3rd,4th,5th,6th,8th Floors \\
\hline Channel Cable Tray & $300 \mathrm{~mm}$ & $100 \mathrm{~mm}$ & 35.9 & & 2nd,3th,4th,5th,6th,8th Floors \\
\hline Channel Cable Tray & $300 \mathrm{~mm}$ & $100 \mathrm{~mm}$ & 35.9 & & 2nd,3rd,4th,5th,6th,8th Floors \\
\hline Subtotal & & & $\mathbf{2 1 5 . 4}$ & & \\
\hline Channel Cable Tray & $300 \mathrm{~mm}$ & $100 \mathrm{~mm}$ & 38.2 & & 7th Floor \\
\hline Subtotal & & & 38.2 & & \\
\hline Channel Cable Tray & $300 \mathrm{~mm}$ & $100 \mathrm{~mm}$ & 1.27 & & Parking Floor \\
\hline Channel Cable Tray & $300 \mathrm{~mm}$ & $100 \mathrm{~mm}$ & 3.33 & & Parking Floor \\
\hline Channel Cable Tray & $300 \mathrm{~mm}$ & $100 \mathrm{~mm}$ & 17.87 & & Parking Floor \\
\hline Subtotal & & & $\mathbf{2 2 . 4 7}$ & & \\
\hline
\end{tabular}

\subsubsection{Results of Application of BIM 5D}

Following the type and character of work or material in this study, some items in the BoQ produce similar calculations between manual calculations and BIM 5D, for example, light points, socket outlets, light switches, and others. The following table compares the manual BoQ and 5D BIM BoQ calculations. 
Table 4. Manual BoQ recapitulation

\begin{tabular}{|c|c|c|c|c|c|c|c|c|}
\hline No & Description & Volume & Unit & Unit Rate (IDR) & \multicolumn{2}{|c|}{ Total (IDR) } & \multicolumn{2}{|c|}{ Percentage } \\
\hline 1 & Preparation Works & 960.00 & $\mathrm{~m} 2$ & $1,151,000$ & $1,104,960,000$ & & & \\
\hline \multirow{2}{*}{2} & Preliminaries/Project Management & 12.00 & months & $700,000,000$ & $8,400,000,000$ & & & \\
\hline & & & & & Sub Total & $9,504,960,000$ & 9.049 & $\%$ \\
\hline 3 & Pile & $3,700.00$ & $\mathrm{~m}^{\prime}$ & 550,000 & $2,035,000,000$ & & & \\
\hline 4 & Foundation & 690.00 & $\mathrm{~m} 2$ & $3,500,000$ & $2,415,000,000$ & & & \\
\hline \multirow[t]{2}{*}{5} & Semi Basement + Gate Works & $1,056.00$ & $\mathrm{~m} 2$ & $2,500,000$ & $2,640,000,000$ & & & \\
\hline & & & & & Sub Total & $7,090,000,000$ & 6.750 & $\%$ \\
\hline \multirow[t]{2}{*}{6} & Upper Structure & $6,200.00$ & $\mathrm{~m} 2$ & $2,550,000$ & $15,810,000,000$ & & & \\
\hline & & & & & Sub Total & $15,810,000,000$ & 15.052 & $\%$ \\
\hline 7 & Wall Finishes & $13,000.00$ & $\mathrm{~m} 2$ & 400,000 & $5,200,000,000$ & & & \\
\hline 8 & Floor Finishes & $9,200.00$ & $\mathrm{~m} 2$ & 475,000 & $4,370,000,000$ & & & \\
\hline 9 & Ceiling Finishes & $6,200.00$ & $\mathrm{~m} 2$ & 300,000 & $1,860,000,000$ & & & \\
\hline 10 & Door and Window Works + Hardware & $5,500.00$ & $\mathrm{~m} 2$ & $1,700,000$ & $9,350,000,000$ & & & \\
\hline 11 & Stair and railing & 480.00 & $\mathrm{~m} 2$ & $2,200,000$ & $1,056,000,000$ & & & \\
\hline \multirow[t]{2}{*}{12} & Sanitair & 162.00 & set & $7,500,000$ & $1,215,000,000$ & & & \\
\hline & & & & & Sub Total & $23,051,000,000$ & 21.945 & $\%$ \\
\hline 13 & Mechanical & $5,500.00$ & $\mathrm{~m} 2$ & $1,485.550$ & $8,170 ., 525,000$ & & & \\
\hline 14 & Electrical & $5,500.00$ & $\mathrm{~m} 2$ & 2.094 .450 & 11.519 .47 .5000 & & & \\
\hline \multirow[t]{2}{*}{15} & Plumbing & $5,500.00$ & $\mathrm{~m} 2$ & $1,722.950$ & $9,476,225,000$ & & & \\
\hline & & & & & Sub Total & $26,675,000,000$ & 27,767 & $\%$ \\
\hline 16 & Interior & $5,500.00$ & $\mathrm{~m} 2$ & $2,000,000$ & $11,000,000,000$ & & & \\
\hline 17 & Landscape & 300.00 & $\mathrm{~m} 2$ & 800,000 & $240,000,000$ & & & \\
\hline \multirow[t]{2}{*}{18} & Lift & 1.00 & unit & $975,000,000$ & $975,000,000$ & & & \\
\hline & & & & & Sub Total & $12,215,000,000$ & 11.629 & $\%$ \\
\hline 19 & Generator/Set & 500.00 & kva & $1,950,000$ & $975,000,000$ & & & \\
\hline 20 & Power House & 500.00 & kva & 900,000 & $450,000,000$ & & & \\
\hline \multirow[t]{2}{*}{21} & Lighting Supply & 800.00 & set & 500,000 & $400,000,000$ & & & \\
\hline & & & & & Sub Total & $1,825,000,000$ & 1.78 & $\%$ \\
\hline \multirow[t]{3}{*}{22} & Façade (Frame + ACP) & $1,594.00$ & $\mathrm{~m} 2$ & $4,000,000$ & $6,376,000,000$ & & & \\
\hline & & & & & Sub Total & $6,376,000,000$ & 6.070 & $\%$ \\
\hline & & & & TOTAL & $105, \mathbf{0 3 8}, \mathbf{1 8 5 , 0 0 0}$ & $105.038,185.000$ & 100.000 & $\%$ \\
\hline
\end{tabular}

Table 5. BoQ using BIM 5 D (Revit)

\begin{tabular}{|c|c|c|c|c|c|c|c|}
\hline \multirow{2}{*}{$\begin{array}{c}\text { No } \\
1\end{array}$} & \multirow{2}{*}{$\begin{array}{r}\text { Description } \\
\text { Preparation Works }\end{array}$} & \multirow{2}{*}{$\begin{array}{r}\text { Volume } \\
960.00\end{array}$} & \multirow{2}{*}{$\frac{\text { Unit }}{\mathrm{m} 2}$} & \multirow{2}{*}{$\begin{array}{c}\text { Unit Rate (IDR) } \\
1,151,000\end{array}$} & \multicolumn{2}{|c|}{ Total (IDR) } & Percentage \\
\hline & & & & & $1,104,960,000$ & & \\
\hline \multirow[t]{2}{*}{2} & Preliminaries/ Project Management & 12.00 & bln & $700,000,000$ & $8,400,000,000$ & & \\
\hline & & & & & Sub Total & $9,504,960,000$ & 9.269 \\
\hline 3 & Pile & $3,700.00$ & $\mathrm{~m}^{\prime}$ & 550,000 & $2,035,000,000$ & & \\
\hline 4 & Foundation & 690.00 & $\mathrm{~m} 2$ & $3,500,000$ & $2,415,000,000$ & & \\
\hline \multirow[t]{2}{*}{5} & Semi Basement + Gate Works & $1,056.00$ & $\mathrm{~m} 2$ & $2,500,000$ & $2,640,000,000$ & & \\
\hline & & & & & Sub Total & $7,090,000,000$ & 6.914 \\
\hline \multirow[t]{2}{*}{6} & Upper Structure & $6,200.00$ & $\mathrm{~m} 2$ & $2,550,000$ & $15,810,000,000$ & & \\
\hline & & & & & Sub Total & $15,810,000,000$ & 15.417 \\
\hline 7 & Wall Finishes & $13,000.00$ & $\mathrm{~m} 2$ & 400,000 & $5,200,000,000$ & & \\
\hline 8 & Floor Finishes & $9,200.00$ & $\mathrm{~m} 2$ & 475,000 & $4,370,000,000$ & & \\
\hline 9 & Ceiling Finishes & $6,200.00$ & $\mathrm{~m} 2$ & 300,000 & $1,860,000,000$ & & \\
\hline 10 & Door and Window Works + Hardware & $5,500.00$ & $\mathrm{~m} 2$ & $1,700,000$ & $9,350,000,000$ & & \\
\hline 11 & Stair and railing & 480.00 & $\mathrm{~m} 2$ & $2,200,000$ & $1,056,000,000$ & & \\
\hline \multirow[t]{2}{*}{12} & Sanitair & 162.00 & set & $7,500,000$ & $1,215,000,000$ & & \\
\hline & & & & & Sub Total & $23,051,000,000$ & 22.478 \\
\hline 13 & Mechanical & $5,500.00$ & $\mathrm{~m} 2$ & $1,650,000$ & $9,075,000,000$ & & \\
\hline 14 & Electrical & $5,500.00$ & $\mathrm{~m} 2$ & $1,450,000$ & $7,975,000,000$ & & \\
\hline \multirow[t]{2}{*}{15} & Plumbing & $5,500.00$ & $\mathrm{~m} 2$ & $1,750,000$ & $9,625,000,000$ & & \\
\hline & & & & & Sub Total & $26,675,000,000$ & 26.012 \\
\hline 16 & Interior & $5,500.00$ & $\mathrm{~m} 2$ & $2,000,000$ & $11,000,000,000$ & & \\
\hline 17 & Landscape & 300.00 & $\mathrm{~m} 2$ & 800,000 & $240,000,000$ & & \\
\hline \multirow[t]{2}{*}{18} & Lift & 1.00 & unit & $975,000,000$ & $975,000,000$ & & \\
\hline & & & & & Sub Total & $12,215,000,000$ & 11.912 \\
\hline 19 & Generator Supply & 500.00 & kva & $1,950,000$ & $975,000,000$ & & \\
\hline 20 & Power House & 500.00 & kva & 900,000 & $450,000,000$ & & \\
\hline \multirow[t]{2}{*}{21} & Lighting Supply & 800.00 & set & 500,000 & $400,000,000$ & & \\
\hline & & & & & Sub Total & $1,825,000,000$ & 1.780 \\
\hline \multirow[t]{3}{*}{22} & ACP Cladding Works & $1,594.00$ & $\mathrm{~m} 2$ & $4,000,000$ & $6,376,000,000$ & & \\
\hline & & & & & Sub Total & $6,376,000,000$ & $6.218 \%$ \\
\hline & & & & JUMLAH & $102,546,960,000$ & $102,546,960,000$ & $100.000 \%$ \\
\hline
\end{tabular}


The BIM 5D process argued takes a shorter time compared to the manual. These findings confirmed the BIM calculation with a quarter to a fifth of the manual calculation time (Bečvarovská \& Matějka, 2014). Besides accelerating the calculation time, BIM 5D also produces better accuracy as seen in the comparison results. The findings also show that BIM 5D provides a more efficient result of $3.56 \%$. The comparison can be seen in the following table.

Table 6. Comparison of BoQ Recapitulation Manual vs BIM 5D (Revit)

\begin{tabular}{|c|c|c|c|c|c|c|c|c|c|c|}
\hline \multirow{3}{*}{$\begin{array}{c}\text { No } \\
\mathrm{I} \\
\end{array}$} & \multirow{3}{*}{\begin{tabular}{|c|} 
DESCRIPTION \\
PRELIMINARIES \\
\end{tabular}} & \multirow{3}{*}{$\begin{array}{c}\text { SCOPE OF WORK } \\
\text { UMUM } \\
\end{array}$} & \multicolumn{4}{|c|}{ BUDGET PLAN } & \multicolumn{4}{|c|}{ REVIT 2018} \\
\hline & & & \multirow{2}{*}{$\mathrm{Rp}$} & \multirow[t]{2}{*}{ TOTAL } & \multicolumn{2}{|c|}{$\begin{array}{c}\text { PERCENTAGE } \\
(\%)\end{array}$} & \multirow[b]{2}{*}{$\mathrm{Rp}$} & \multirow[t]{2}{*}{ TOTAL } & \multicolumn{2}{|c|}{$\begin{array}{c}\text { PERCENTAGE } \\
(\%)\end{array}$} \\
\hline & & & & & & & & & & \\
\hline & & & & & & & & & & \\
\hline II & LIGHTING WORKS & MECHANICAL & $\mathrm{Rp}$ & $4,689,054,400$ & & & $\mathrm{Rp}$ & $4,286,145,400$ & & \\
\hline III & CABEL TRAY WORKS & MECHANICAL & $\mathrm{Rp}$ & $1,097,847,200$ & & & $\mathrm{Rp}$ & $854,654,800$ & & \\
\hline IV & LIGHTNING ROD WORKS & MECHANICAL & $\mathrm{Rp}$ & $92,107,000$ & & & $\mathrm{Rp}$ & $92,107,000$ & & \\
\hline \multirow[t]{2}{*}{$v$} & POWER PANEL WORKS & MECHANICAL & $\mathrm{Rp}$ & $2,291,478,200$ & & & $\mathrm{Rp}$ & $2,123,453,700$ & & \\
\hline & \multicolumn{2}{|c|}{ SUB TOTAL MECHANICAL WORKS } & $\mathrm{Rp}$ & $8,170,486,800$ & 7,78 & $\%$ & $\mathrm{Rp}$ & $7,356,360,900$ & 7,02 & $\%$ \\
\hline $\mathrm{VI}$ & CCTV WORKS & ELECTRICAL & $\mathrm{Rp}$ & $632,481,400$ & & & $\mathrm{Rp}$ & $370,213,400$ & & \\
\hline VII & SOUND SYSTEM WORKS & ELECTRICAL & $\mathrm{Rp}$ & $99,451,100$ & & & $\mathrm{Rp}$ & $99,451,100$ & & \\
\hline VIII & PABX WORKS & ELECTRICAL & $\mathrm{Rp}$ & $878,087,500$ & & & $\mathrm{Rp}$ & $878,087,500$ & & \\
\hline IX & MATV WORKS & ELECTRICAL & $\mathrm{Rp}$ & $2,752,987,600$ & & & $\mathrm{Rp}$ & $2,645,850,600$ & & \\
\hline $\mathrm{x}$ & ALARM WORKS & ELECTRICAL & $\mathrm{Rp}$ & $2,358,847,100$ & & & $\mathrm{Rp}$ & $2,358,847,100$ & & \\
\hline \multirow[t]{2}{*}{$\mathrm{XI}$} & HVAC WORKS & ELECTRICAL & $\mathrm{Rp}$ & $4,797,531,000$ & & & $\mathrm{Rp}$ & $3,114,137,300$ & & \\
\hline & \multicolumn{2}{|c|}{ SUB TOTAL ELECTRICAL WORKS } & $\mathrm{Rp}$ & $11,519,385,700$ & 10,97 & $\%$ & $\mathrm{Rp}$ & $9,466,587,000$ & 9,01 & $\%$ \\
\hline XII & HYDRANT WORKS & PLUMBING & $\mathrm{Rp}$ & $4,797,531,000$ & & & $\mathrm{Rp}$ & $4,366,114,700$ & & \\
\hline XII & PLUMBING WORKS & PLUMBING & $\mathrm{Rp}$ & $5,678,543,600$ & & & $\mathrm{Rp}$ & $4,957,948,200$ & & \\
\hline \multicolumn{3}{|c|}{ SUB TOTAL PLUMBING WORKS } & $\mathrm{Rp}$ & $9,476,474,600$ & 9,02 & $\%$ & $\mathrm{Rp}$ & $9,324,062,900$ & 8,18 & $\%$ \\
\hline & & GRAND TOTAL & $\mathrm{Rp}$ & $29,166,347,100$ & 27,77 & $\%$ & $\mathrm{Rp}$ & $26,147,010,800$ & 24,21 & $\%$ \\
\hline \multicolumn{11}{|c|}{ DIFFERENCES $=27,77 \%-24,21 \%=3,56 \%$} \\
\hline \multicolumn{11}{|c|}{ BASED ON BIM 5D REVIT EFFICIENCY (3,56 \%) } \\
\hline
\end{tabular}

\section{CONCLUSION}

BIM 5D plays a significant role in providing higher accuracy and faster calculation for building planning and development. The factors that affect the success of BIM 5D implementation consist of 2D drawings, BIM 5D implementation, specification, and technical plan, operator experiences, BIM 5D models, individual selection model, estimating, calculation process, cost database, and operator education. For the cases of MEP work in high-rise hotel buildings, the difference between manual calculations and BIM 5D Revit in terms of cost efficiency is $3.56 \%$.

Despite this result, there is some limitation that can be used to generate more comprehensive result in the future. First, the case study only uses of type of building of high-rise hotels which may differ from other types of building such as residential or commercial buildings. Second, there is some combination of BIM and other advanced technology such as machine learning, IoT, and many others. Adopting this combination may provide more comprehensive findings and significantly improve the results. Last, the case study conducted in developing countries in South East Asia, a similar project in other developed economies or developing countries may beneficial to compare each finding and obtain significant knowledge expansion.

\section{ACKNOWLEDGEMENT}

This research supported by PT. Panton Graha Steel Pattern Building Contractor and PT. Stadia Tujuh Engineering (MEP Contractor).

\section{REFERENCES}

Abu Hammad, A. A., Ali, S. M. A., Sweis, G. J., \& Sweis, R. J. (2010). Statistical Analysis on the Cost and Duration of Public Building Projects. Journal of Management in Engineering, 26(2), 105-112. https://doi.org/10.1061/(asce)0742-597x(2010)26:2(105)

Akhil, R. P., \& Das, B. B. (2019). Cost reduction techniques on MEP projects. In Lecture Notes 
in Civil Engineering (Vol. 25, pp. 495-517). https://doi.org/10.1007/978-981-13-3317-0_44 Alaloul, W. S., Liew, M. S., \& Zawawi, N. A. W. A. (2016). Identification of coordination factors affecting building projects performance. Alexandria Engineering Journal, 55(3), 26892698. https://doi.org/10.1016/j.aej.2016.06.010

Alrashed, I., \& Kantamaneni, K. (2018). A 5D building information model (BIM) for potential cost-benefit housing: A case of Kingdom of Saudi Arabia (KSA). Infrastructures, 3(2). https://doi.org/10.3390/infrastructures3020013

Aneziris, O. N., Topali, E., \& Papazoglou, I. A. (2012). Occupational risk of building construction. Reliability Engineering and System Safety, 105, 36-46. https://doi.org/10.1016/j.ress.2011.11.003

Azhar, S., Carlton, W. A., Olsen, D., \& Ahmad, I. (2011). Building information modeling for sustainable design and LEED ${ }^{\circledR}$ rating analysis. Automation in Construction, 20(2), $217-$ 224. https://doi.org/10.1016/j.autcon.2010.09.019

Barlish, K., \& Sullivan, K. (2012). How to measure the benefits of BIM - A case study approach. Automation in Construction, 24, 149-159. https://doi.org/10.1016/j.autcon.2012.02.008

Bečvarovská, R., \& Matějka, P. (2014). Comparative Analysis of Creating Traditional Quantity Takeoff Method and Using a BIM Tool. Construction Maeconomics Conference, 1-4. http://www.conference-cm.com/podklady/history5/Prispevky/paper_becvarovska.pdf

Bynum, P., Issa, R. R. A., \& Olbina, S. (2013). Building Information Modeling in Support of Sustainable Design and Construction. Journal of Construction Engineering and Management, 139(1), 24-34. https://doi.org/10.1061/(asce)co.1943-7862.0000560

Charef, R., Alaka, H., \& Emmitt, S. (2018). Beyond the third dimension of BIM: A systematic review of literature and assessment of professional views. Journal of Building Engineering, 19, 242-257. https://doi.org/10.1016/j.jobe.2018.04.028

Gündüz, M., Nielsen, Y., \& Özdemir, M. (2013). Quantification of Delay Factors Using the Relative Importance Index Method for Construction Projects in Turkey. Journal of Management in Engineering, 29(2), 133-139. https://doi.org/10.1061/(asce)me.19435479.0000129

Husin, A. E., Fahmi, F., Rahardjo, S., Siregar, I. P., \& Kussumardianadewi, B. D. (2019). MPERT and lean construction integration on steel construction works of warehouse buildings. International Journal of Engineering and Advanced Technology, 8(4), 696-702.

Husin, A. E., Setyawan, T. L., Meidiyanto, H., Kussumardianadewi, B. D., \& Eddy Husin, M. K. (2019). Key success factors implementing BIM based quantity take-off in fit-out office work using relative importance index. International Journal of Engineering and Advanced Technology, 8(6), 986-990. https://doi.org/10.35940/ijeat.F82650.88619

Kim, S., Chin, S., \& Kwon, S. (2019). A Discrepancy Analysis of BIM-Based Quantity Take-Off for Building Interior Components. Journal of Management in Engineering, 35(3), 05019001. https://doi.org/10.1061/(asce)me.1943-5479.0000684

Riley, D. R., Varadan, P., James, J. S., \& Thomas, H. R. (2005). Benefit-Cost Metrics for Design Coordination of Mechanical, Electrical, and Plumbing Systems in Multistory Buildings. Journal of Construction Engineering and Management, 131(8), 877-889. https://doi.org/10.1061/(asce)0733-9364(2005)131:8(877)

Staub-French, S., Fischer, M., Kunz, J., \& Paulson, B. (2003). An Ontology for Relating Features with Activities to Calculate Costs. Journal of Computing in Civil Engineering, 17(4), 243254. https://doi.org/10.1061/(asce)0887-3801(2003)17:4(243)

Volk, R., Stengel, J., \& Schultmann, F. (2014). Building Information Modeling (BIM) for existing buildings - Literature review and future needs. In Automation in Construction (Vol. 38, pp. 109-127). https://doi.org/10.1016/j.autcon.2013.10.023

Wang, L., \& Leite, F. (2016). Formalized knowledge representation for spatial conflict coordination of mechanical, electrical and plumbing (MEP) systems in new building 

In Hotel Based on Building Information Modeling (BIM) 5D

projects. Automation in Construction, 20-26. https://doi.org/10.1016/j.autcon.2015.12.020 